\title{
Artificial neural network based meta-heuristic for performance improvement in physical internet supply chain network
}

\author{
Chouar Abdelsamad ${ }^{1}$, Tetouani Samir ${ }^{2}$, Soulhi Aziz ${ }^{3}$, Elalami Jamila ${ }^{4}$ \\ ${ }^{1,2}$ Laboratoire d'Analyse des Systèmes, Traitement de l'Information et Management Intégré (LASTIMI), Université \\ Mohammed V-Agdal Ecole Mohammadia d'Ingénieurs, Rabat, Morocco \\ ${ }^{1,2}$ Centre d'Excellence en Logistique (CELOG), École Supérieure de l'industrie du Textile et d'Habillement (ESITH), \\ Casablanca, Morocco \\ ${ }^{3}$ Superior National School of Mines, Rabat, Morocco \\ ${ }^{4}$ National Center for Scientific and Technical Research (CNRST), Rabat, Morocco
}

\begin{abstract}
Article Info
Article history:

Received Jun 3, 2021

Revised Sep 11, 2021

Accepted Sep 15, 2021

\section{Keywords:}

Artificial neural networks

Slime mould algorithm

Supply chain management Physical internet

ABSTRACT

Nowadays, reducing total costs while enhancing customer satisfaction is a major task for many supply chain systems. To deal with this issue, the physical internet (PI) paradigm can be represented as a potential replacement for the current logistics system. This paper devoted the cost reduction and lead time improvement in a PI-SCN using a hybrid framework based on an artificial neural network (ANN) and an improved slime mould algorithm (ISMA). To address the performance of the proposed framework, a real-case study in Morocco is considered. The new trainer ISMA's performance has been investigated in three approximation datasets from the University of California at Irvine (UCI) machine-learning repository regarding nine recent metaheuristics. The experimental results highlight the effectiveness of ISMA according to other meta heuristics for training feed-forward neural networks (FNNs) to converge speed and to avoid local minima.
\end{abstract}

This is an open access article under the CC BY-SA license.

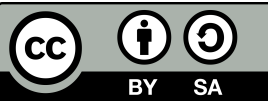

\section{Corresponding Author:}

Chouar Abdelsamad

Centre d'Excellence en Logistique (CELOG)

École Supérieure de l'industrie du Textile et d'Habillement (ESITH)

Casablanca, Morocco

Email: chouar@esith.ac.ma

\section{INTRODUCTION}

Nowadays, the major strength for the global logistics operations is to ensure a sustainable systems through integrating the developed technologies and methodologies to the real world practices. For a large logistics scale, the logistics web aims to connect the supply chain's network including the different actors, physical items and digital technologies in order to assist the global requirements. From a broadly perspective, the physical internet (PI) aims to optimize the supply chain processes according to a defined framework to enhance the logistics web efficiency, effectiveness and sustainability which develop the required reliability, resilience and adaptability. The purpose of the innovative Physical Internet (PI or $\pi$ ) initiative is to reverse the situation of existing unsustainable in current logistics systems. Indeed, due to the dynamic nature of real-world problems, logistics web design models must take into consideration the risks of disruption and unforeseen events to ensure the resilience and efficiency of the entire logistics web chain. For example, taking into account 
uncertainties (demand and road traffic) and assessing the risks of disruptions caused by major crises, such as the crisis of the coronavirus disease (COVID-19) epidemic.

The capacity to measure the strategical, tactical and operational performance is considered as a main framework to assert inevitably in order to strengthen the enterprises competitiveness within physical internet supply chain networks. This allows the long-term outputs effects assessment to further support the competitiveness and decision-making power [1]. Therefore, a well-defined set of performance indicators is mandatory to consolidate the required objectives for the overall performance measurement. The underpinning to uncover these indicators in order to increase the chances of success is an overall analysis of the company's environment processes [2]. Thus, correction's advantages are performed through evaluation perspectives which must gather financial and non-financial measures. Since the presence of many criteria, the performance measurement in physical internet supply chain network (PISCN) is defined as a problem which belongs to multiple criteria decision making. In fact, multiple methodologies have been developed to evaluate a multiple criteria scheme such as data envelopment analysis (DEA) [3]. Accordingly, the DEA contributes to assess the efficiency surfaces through a mathematical programming model. Whereas, the resolution process can be affected statistical noises which extent to wrap the derived frontier [4].

Due to the panoply of choice for decision-making processes, the non-parametric tool for non-linear relations between inputs and outputs modeling approach has been widely developed with the artificial neural network (ANN) [5]. In fact, the ANN presents a wide variety in the literature such as spiking neural networks [6] and recurrent neural networks [7], though, the most popular type is the feed-forward neural networks (FNNs) [8]. Besides, the learning process (i.e., training process) has a huge impact on the process performance of ANNs. As a whole, training algorithms can be arranged into two categories: gradient-based algorithms versus stochastic search algorithms. It can be noted from [9] that the widely adopted gradient-based training algorithm is back-propagation (BP). To some extent, the cons of this method are evinced, for instance, through the tardy convergence behavior and hanging on local minima.

Besides, for optimization problems, considering some nature-inspired metaheuristics algorithms as alternative trainers have proved a higher efficiency to diverge from local minima. The large potential of metaheuristic methods to train the feed-forward neural networks (FNNs) has been widely asserted in the literature. In this respect, the krill herd algorithm (KHA) has been established for data classification to train the FNNs [10]. Not long ago in 2016, a nature-inspired algorithm known as multiverse optimizer (MVO) has been used for training the FNNs [11]. It is worth mentioning that through the reported numerical results, the MVO reveals a high competitiveness and it performs better than another training algorithms in most of datasets.

In spite of the high quality of the previous presented works, the local optima entrapment's issue continues to be faced. Besides, as mentioned by [12], a theorem known as No Free Lunch within the heuristics area highlights the lack of a generic problem solving optimization algorithm. Given that, the performance gap between algorithms occurs after FNNs training for multiple data sets. Thus, new algorithms efficiencies for learning FNNs are considered as a worthy field to be addressed by researchers. In this respect, this paper aims to embed the newly slime mould algorithm (SMA) algorithm [13] into FNNs.

This paper appraises the physical internet supply chain network performance (PI-SCN). The proposed structre is two steps based. Firstly, we depict three features' categories (i.e., economic, social, and environmental) in addition to the target variables (reducing costs and lead time improvement) affecting the physical internet supply chain network (PI-SCN). Secondly and in order to train the FNNs, a new method formulation has been applied using the improved slime mould algorithm (ISMA) to reach the efficiency values.

The remainder of this article is organized as follows. The performance measurement system is discussed in the section 2. Section 3 details the methodologies applied in this study. Section 4 presents numerical computations and discussion. At the end, section 5 summarizes the conclusions and points out future researches.

\section{PHYSICAL INTERNET SUPPLY CHAIN NETWORK (PI-SCN) PERFORMANCE SYSTEM}

The assessment of operations' performance in a physical internet supply chain network stands among the main managerial business affair. But, it is very tough to evaluate an organization's performance when several measures belongs to a defined system or operation [14]. Besides, the expanding competitiveness in the supply chain domain requires more advanced performance level. According to the global objectives of the company, the related system of performance measurement's indicator will be drawn [15]. The importance 
of financial measures to assess the organizations' profits point out their existence within many performance measurement frameworks proposed for physical supply chain network. This study highlights the objectives to rely on as well as the involved performance indicators to reach the targeted PI-SCN performances. The whole steps are presented in Figure 1.

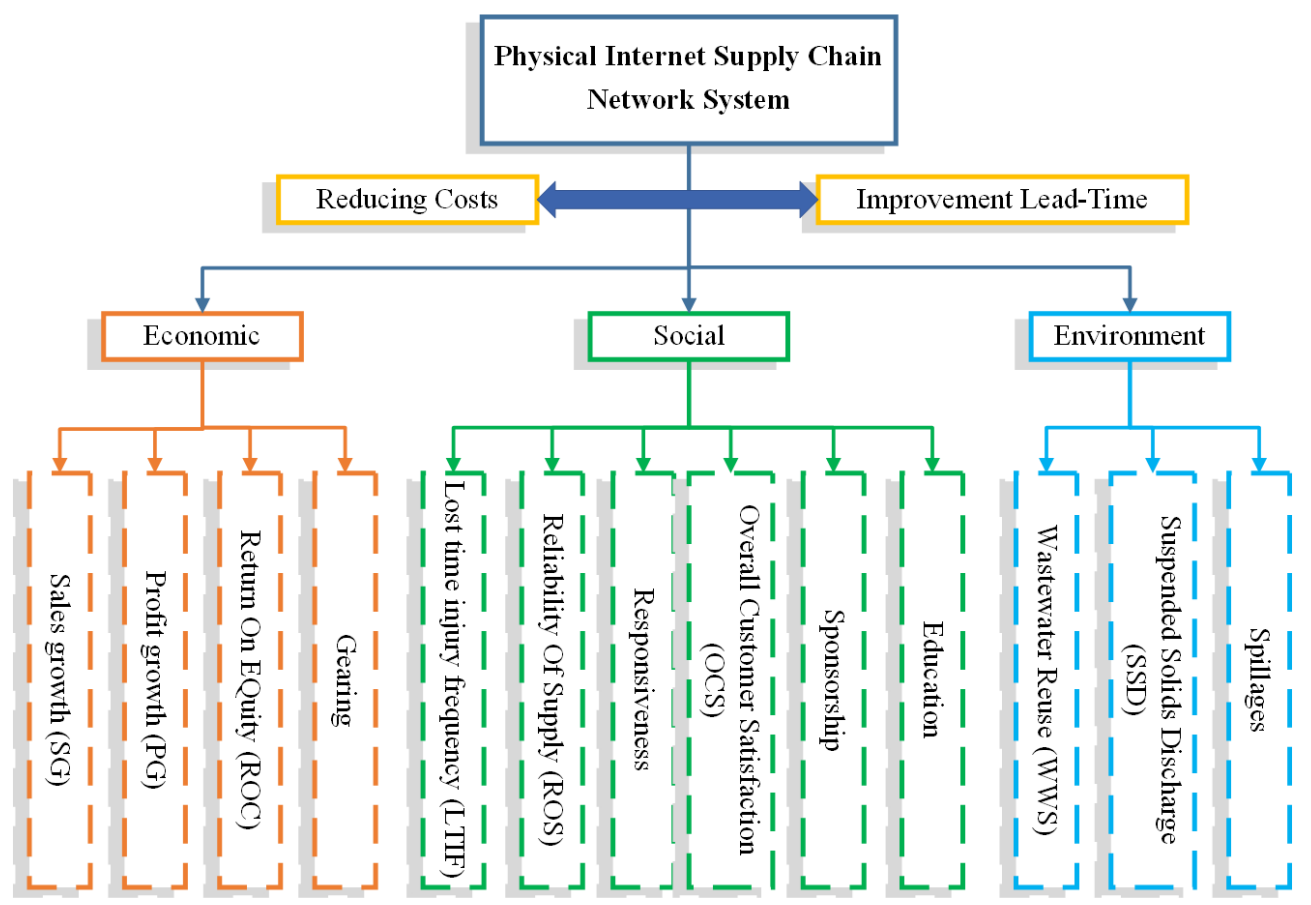

Figure 1. Physical internet supply chain network system

\section{RESEARCH METHOD}

In this part, we illustrate the recommended framework to evaluate the performance of (PI-SCN).

\subsection{Feed-forward neural network}

One of the most popular type of ANNs is the FNNs. In this network, the information has a unique progression's direction which start from the inputs to outputs by moving across set "neurons" [16] in hidden layer. Nevertheless, the network does not intend any cycles or loops. The Figure 2 illustrates an elementary FNN with a single hidden layer. As highlighted, the sum of the inputs' weight are computed by each neuron considering a bias. Subsequently, the sum is passed across sigmoid function and then reach the output of NN. The procedure is represented by (1)-(3):

$$
H_{j}=\sum_{i=1}^{R} \omega_{i, j} * I_{j}+b_{j}
$$

Where $R$ is the number of nodes of input layers, $\omega_{i, j}$ denotes the connection weight between the $i^{\text {th }}$ neuron of the input layer and $j^{t h}$ neuron of the hidden layer, $b_{j}$ is the threshold (bias) in hidden layers and $I_{i}$ is the $i^{t h}$ input data.

$$
f(x)=\frac{1}{1+e^{-x}}
$$

Here $f_{(x)}$ is the sigmoid function. The output of the network is calculated as follows:

$$
y_{k}=f_{k}\left(\sum_{j=1}^{N} \Gamma_{i, j} * H_{j}+b_{k}\right)
$$


Where $\Gamma_{i, j}$ denotes the connection weight between the $j^{\text {th }}$ neuron of the hidden layer and $k^{\text {th }}$ neuron of the output layer, $b_{k}$ is the threshold (bias) in output layers. As long as some error criterion is not reached, the training procedure is performed to regulate the weights and bias. As a matter of fact, pick up the proper training algorithm is the principal challenge. Moreover, the design complexity of the neural network increases given that many elements affect the training performance, for instance, the total nodes in hidden layers, in addition to the error and activation functions. Figure 2 shows a simple FNN structure.

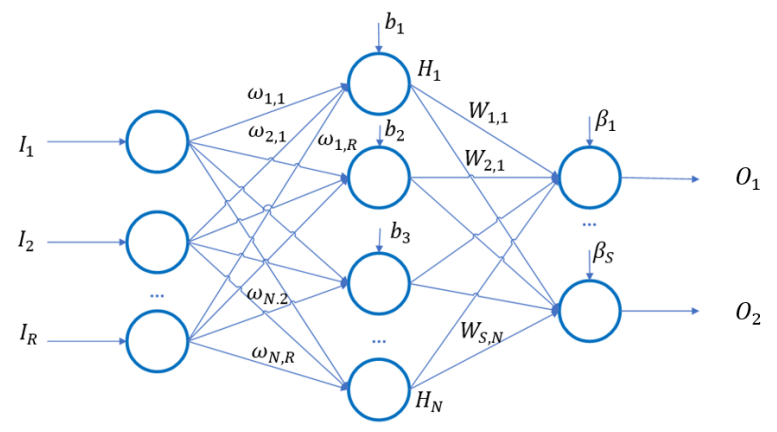

Figure 2. FNN architecture

\subsection{Brief description of SMA}

A newly optimization technique has been presented not long ago by [13] called the SMA, the general concept is simulated from slime moulds, physarum polycephalum intelligent behaviour. Henceforward, this algorithm which is based on a population stochastic search procedure has been employed into many complex engineering problems in the field of optimization. The principal concepts of SMA are outlined in the following subsection.

\subsubsection{Approach food}

To model the approaching behavior of slime mould as a mathematical equation, the following rule is proposed to imitate the contraction mode by using (4):

$$
X_{t+1}=\left\{\begin{array}{cc}
X_{b}(t)+v_{b} \cdot\left(W \cdot X_{A}(t)-X_{B}(t)\right) & r<p \\
v_{c} \cdot X_{t} & r \geq p
\end{array}\right.
$$

where $v_{b}$ is a parameter with a range of $[-a, a], v_{c}$ decreases linearly from one to zero. The $t$ represents the current iteration, $X_{b}$ represents the individual location with the highest odor concentration currently found, $X$ represents the location of slime mould, $X_{A}$ and $X_{B}$ represent two individuals randomly selected from the swarm, $W$ represents the weight of slime mould. The formula of $p$ is as follows using (5):

$$
p=\tanh |S(i)-D F|
$$

where $i \in 1,2, \ldots, n, S(i)$ represents the fitness of $X, D F$ represents the best fitness obtained in all iterations.

The formula of $v_{b}$ is as follows by (6):

$$
v_{b}=[-a, a]
$$

The formula of $a$ is as follows by (7):

$$
a=\operatorname{arctanh}\left(-\left(\frac{t}{\max _{t}}\right)+1\right)
$$

The formula of $W$ is listed as follows by (8):

$$
W(\text { SmellIndex }(i))=\left\{\begin{array}{lc}
1+r \log \left(\left(b_{F}-S(i)\right) /\left(b_{F}-w_{F}\right)+1\right) & \text { condition } \\
1-r \log \left(\left(b_{F}-S(i)\right) /\left(b_{F}-w_{F}\right)+1\right) & \text { others }
\end{array}\right.
$$




$$
\text { SmellIndex }=\operatorname{sort}(S)
$$

where condition indicates that $S(i)$ ranks first half of the population, $r$ denotes the random value in the interval of [0,1], $b_{F}$ and $w_{F}$ illustrates, respectively, the obtained optimal and worst fitnesses in the current iterative process, while SmellIndex denotes the sorted sequence of fitness values (ascends in the minimum value problem).

\subsubsection{Wrap food}

The mathematical formula for updating the location of slime mould is described by (10):

$$
X^{*}=\left\{\begin{array}{cc}
\operatorname{rand}(U B-L B)+L B & \text { rand }<z \\
X_{b}(t)+v_{b}\left(W X_{A}(t)-X_{B}(t)\right) & r<p \\
v_{c} X(t) & r \geq p
\end{array}\right.
$$

where $L B$ and $U B$ outline the lower and upper boundaries of the search range, rand and $\mathrm{r}$ define the random value within $[0.1]$.

\subsubsection{Oscillation}

The value of $v_{b}$ oscillates randomly between $[-a, a]$ and it gradually converges toward zero as well as the iterations increase. The value of $v_{c}$ oscillates between $[-1,1]$ and tends to zero eventually. The main steps of the SMA are illustrated in the figure and the algorithm.

\subsection{Lévy flights}

Levy flights is a non-Gaussian stochastic process, the related step sizes are distributed based on a Levy stable distribution to generate new solutions. Once a novel solution is defined, the following Levy flight is carried out in (11):

$$
X_{t+1}=X_{t} \oplus \operatorname{Levy}(\lambda)
$$

where $\lambda$ indicates the step size related to the problem's scales. The product $\oplus$ means entry-wise multiplications. The prevailing idea is that Levy flights furnish a random walk given that for large steps. In this study, the algorithm proposed by [16] will be used on account of its prominent efficient highlighted with Levy flights implementation.

\subsection{Improved slime mould algorithm}

The prevailing criteria for an efficient optimization algorithm are based on the strong exploration ability in addition to a fast exploitation rate. With the aim for SMA performance's improvement and to expand the algorithm exploration, an update position-based accelerated particle swarm optimization (APSO) [17] and Lévy flight technique are included into the SMA. The principal intention of the suggested algorithm is in that way.

The basic idea of the proposed algorithm is as follows. First, a fraction of the population is chosen according to the worst fitness value. Then a Lévy flight is performed according section 2.2, while the standard SMA is applied to the rest of better solutions. Secondly, an update position-based is implemented based on APSO. Generally, APSO has the ability to prospect rapidly the search space and find out efficiently the optimal solution. Hence, the position is updated by the following (12):

$$
X_{t+1}=(1-\beta) * X_{t}+\beta * g^{*}+\alpha * r
$$

The velocity is not included in the equation 12, thereof, the APSO does not require velocities' initialization, in this regard, it avoids the drawbacks related to regular PSO velocities. At this stage the third term $r$ compels the system to be more mobile and to avoid entrapment within any local optima if its selection has been performed properly, the corresponding definition of $r$ can be drawn from a statistical distribution. According to the other parameters such as $\beta$ and $\alpha$ are choosing according to [17] as follow in (13):

$$
\alpha=\gamma^{t}
$$

where $\beta=0.2-0.7$ and $\gamma=0.1-0.99$. Here $t \in\left[0, t_{\max }\right]$. The proposed method is outlined in Algorithm 1: 


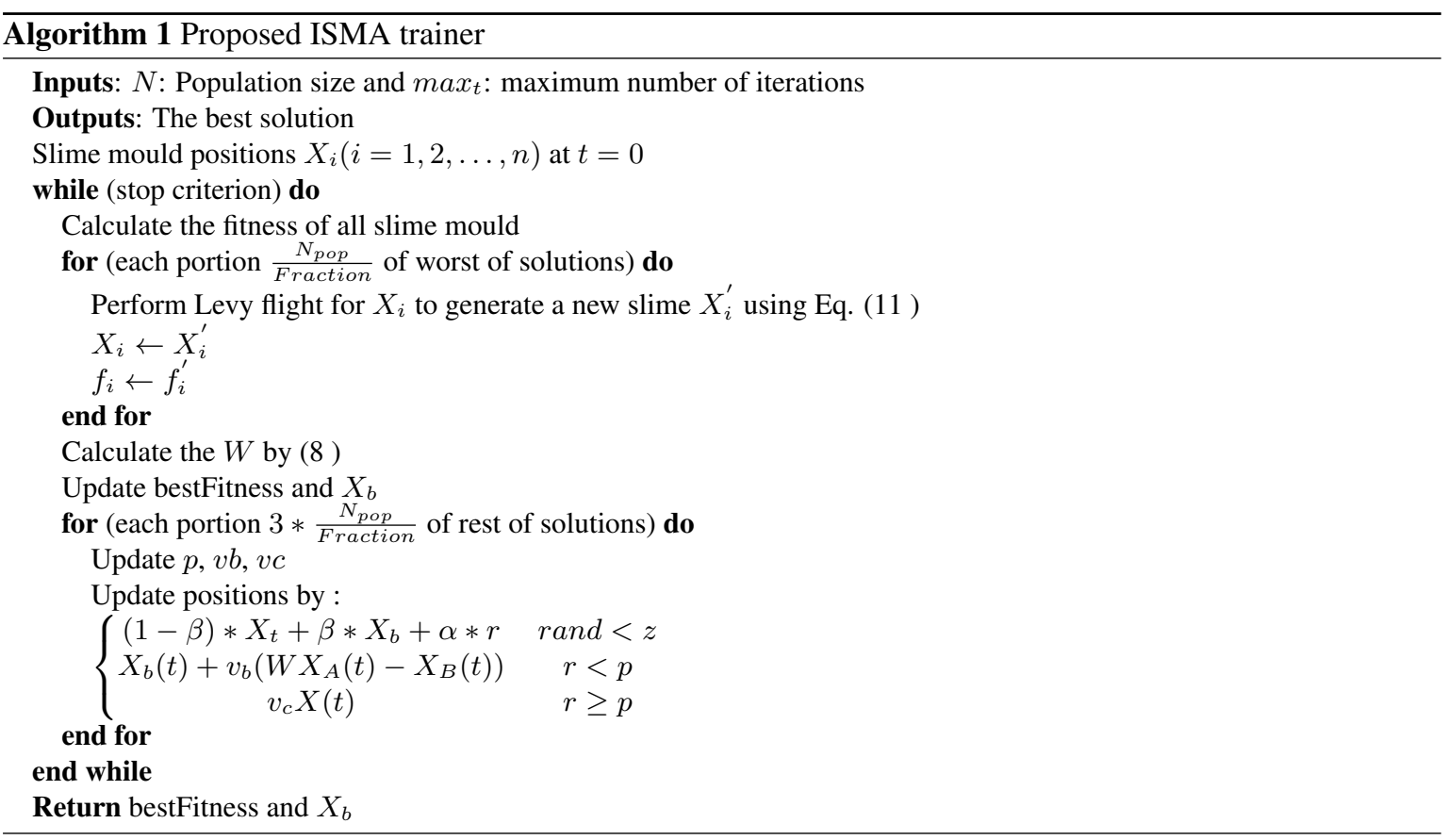

\section{ISMA FOR TRAIN FNNS}

\subsection{Architecture of FNNs}

During NNs employment, the structure should be essentially defined according to the layers' number in addition to layers' neurons number. The NN complexity's is correlated to the number of neurons in the hidden layers. As long as the number is important, the complexity increases. In this study, the characteristic of a problem-dependent has been associated to the input and output neurons' number in MLP network so that the Kolmogorov theorem [18] has been adopted to compute the number of hidden nodes through the (14):

$$
H=2 * I+1
$$

The network's weights and bias have been optimized through SMA. Moreover, D reflects each organism's dimension:

$$
D=(I * H)+(H * O)+H_{\text {bias }}+O_{\text {bias }}
$$

Notice that $I, H$ and $O$ describe respectively the input, hidden and output FNN's neurons. While $H_{\text {bias }}$ and $O_{\text {bias }}$ presents the biases' number and output layers. Figure 3 shows the FNN architecture for PI-SCN.

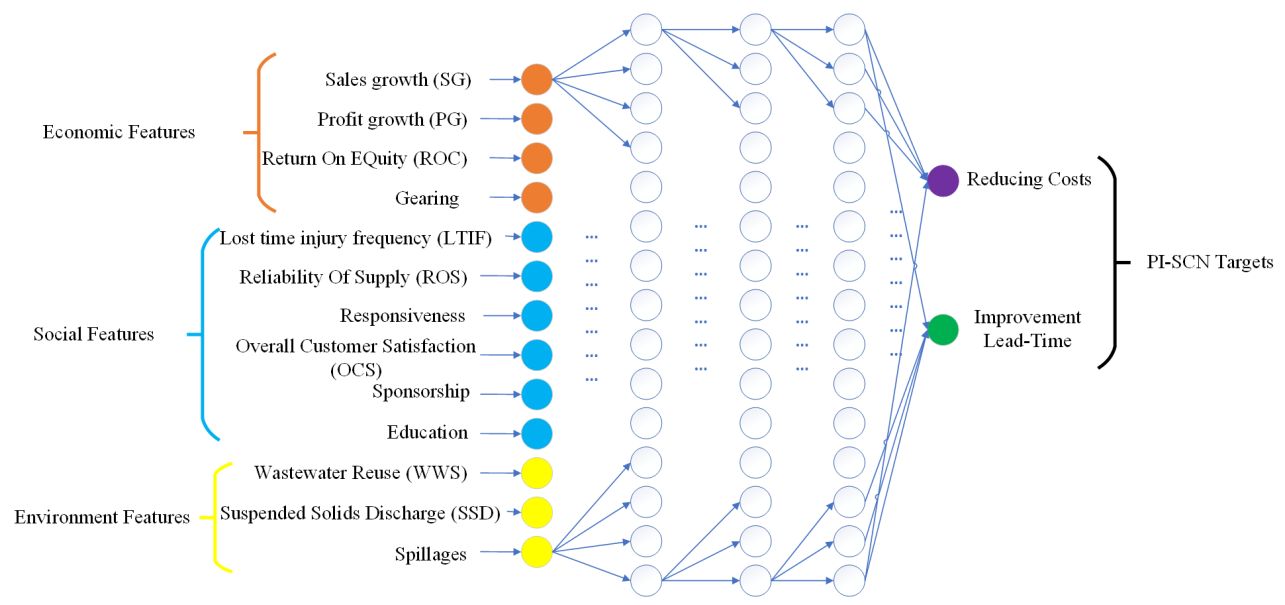

Figure 3. PI-SCN system ANN based representation 


\subsection{Method evaluation}

The evaluation according to ISMA of each slime mould is performed giving its fitness which reflects to its status. The corresponding process is the following; the vector that include the weights and biases is passed to FNNs, afterward, the neural network prediction employing the training dataset is used to figure out the mean squared error (MSE) criterion. The optimal solution is reached after consecutive iterations which describe the neural network weights and biases. The (16) presents the MSE criterion, $M$ describes the samples' number in training dataset and $(\widehat{Y} ; Y)$ are respectiveley to the estimated and the original values according to the suggested model.

$$
M S E=\frac{1}{M} \sum_{i=1}^{M}\left(y_{r}-\widehat{y_{r}}\right)
$$

\subsection{Encoding strategy}

Different encoding strategies have been introduced by [19]. For instance, in the field of evolutionary algorithm, the FNNs' weights and biases for every agent can be structured in multiple forms such as vector, matrix, or binary. The Figure 4 highlights an encoding strategy which belongs to vector structure, this method has been adopted for the present study. In this respect, the FNNs' weights and biases stand for each mould which converted afterwards into a real number single vector.

\begin{tabular}{|l|l|l|l|}
\hline$\omega_{1,1} \ldots \omega_{N, R}$ & $W_{1,1} \ldots W_{S, N}$ & $b_{1} \ldots b_{N}$ & $\beta_{1} \ldots \beta_{S}$ \\
\hline
\end{tabular}

Figure 4. Solution representation

\subsection{Proposed model}

In this part, the suggested model is explored according to a three part. Firstly, three categories of features are identified (i.e., economic, social, and environmental) and the target variables (reducing costs and lead time improvement) that affect our system. At the end, the efficiency scores are determined while implementing ISMA as novel method to train FNNs. The related algorithm for the proposed hybrid framework is reported in the Figure 5.

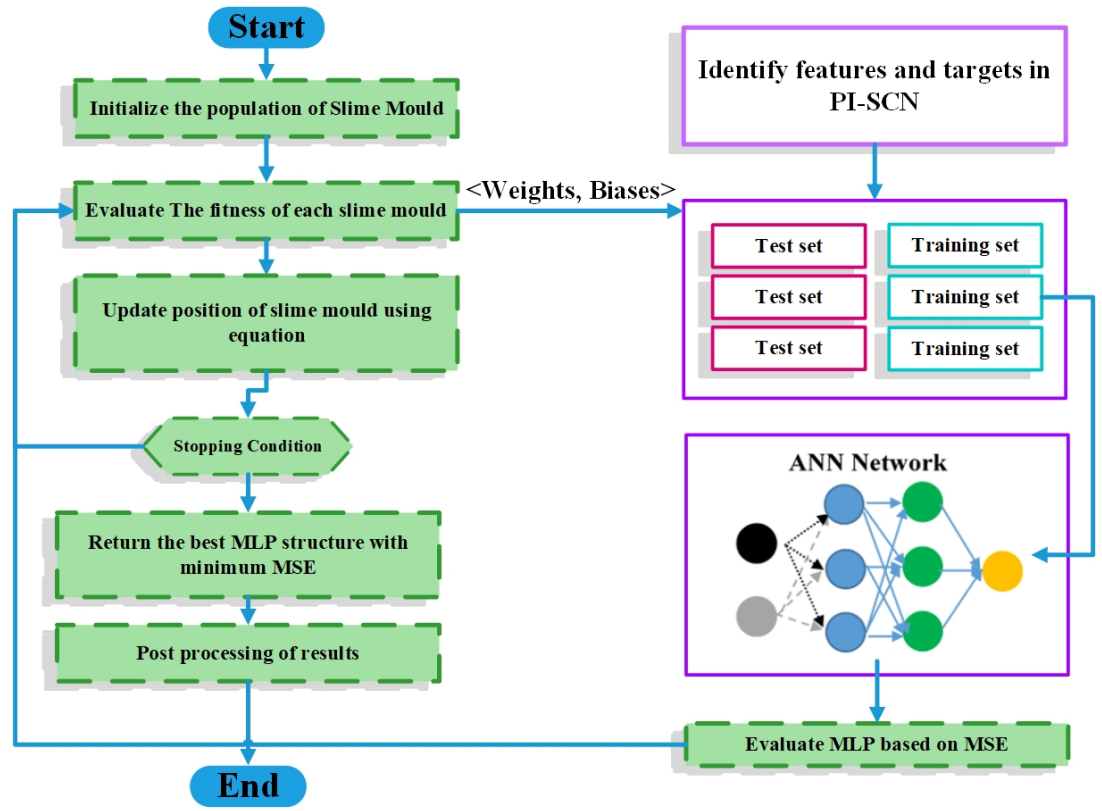

Figure 5. Proposed hybrid framework 


\section{RESULT AND DISCUSSION}

In this part, the suggested hybrid framework efficiency is explored to evaluate the PI-SCN performance. The proposed method is compared against recent nine algorithms such as GA [20], GWO [21], SCA [22], WOA [23], HHO [24], SMA [13], MVO [25], MFO [26]. The whole algorithms were programmed in MATLAB R2014a. The experiments computations have been performed through 20 distinct runs; the other algorithms' parameters are taken the same as the published paper's values. The dataset shown in Table 1 has been gathered exploiting a brainstorming. Because of condientiality concerns, the first data has been changed and partitioned into $66 \%$ for training and $34 \%$ for testing.

Table 1. Textile datasets

\begin{tabular}{|c|c|c|c|c|c|c|c|c|c|c|c|c|c|c|}
\hline \multicolumn{13}{|c|}{ Features } & \multicolumn{2}{|c|}{ Targets } \\
\hline SG & PG & $\mathrm{ROC}$ & $\mathrm{G}$ & LTTF & ROS & $\mathrm{R}$ & OCS & Spo & $\mathrm{E}$ & WWS & SSD & Spi & Costs & LT \\
\hline 10 & 2 & 6 & 6 & 8 & 3 & 6 & 1 & 6 & 2 & 4 & 5 & 4 & 69 & 68 \\
\hline 9 & 6 & 6 & 9 & 1 & 2 & 6 & 3 & 9 & 2 & 5 & 7 & 6 & 76 & 61 \\
\hline 10 & 9 & 6 & 3 & 5 & 2 & 3 & 9 & 1 & 8 & 3 & 5 & 3 & 70 & 53 \\
\hline 6 & 2 & 5 & 10 & 1 & 5 & 2 & 9 & 5 & 1 & 10 & 1 & 6 & 79 & 73 \\
\hline 3 & 2 & 9 & 7 & 9 & 6 & 9 & 4 & 6 & 8 & 6 & 1 & 10 & 66 & 53 \\
\hline 1 & 10 & 10 & 8 & 3 & 7 & 3 & 6 & 3 & 1 & 6 & 6 & 9 & 74 & 73 \\
\hline 7 & 8 & 6 & 5 & 1 & 3 & 8 & 3 & 9 & 8 & 1 & 8 & 5 & 71 & 55 \\
\hline 3 & 10 & 8 & 4 & 9 & 1 & 2 & 1 & 6 & 1 & 3 & 5 & 4 & 51 & 51 \\
\hline
\end{tabular}

In order to compare all algorithms and during all benchmarks, the average (AVE) and the standarg deviation (STD) have been employed. These two mesures have been implemented to highlight the algorithm's effectiveness to escape from local minima entrapment. A case study has been applied based on 100 companies operating in textile industry in order to validate our model. The results of dataset are reported in Table 2. By analysing the results of the Table 2, the prevailing element to share is the best performance outlined by the proposed method as well as MFO and HHO. This behavior is depicted by the highest ability to escape local optima which is fundamentally finer comparing to another algorithms. Besides, a convergence comparative experimentation was implemented to approve that ISMA has greater convergence performance than the other algorithms. Figure 6 shows the convergence curves.

Table 2. Experimental results for textile company dataset

\begin{tabular}{cccccccccc}
\hline & GA & GWO & SCA & WOA & HHO & SMA & MVO & MFO & ISMA \\
\hline Min & $9,86 \mathrm{E}-04$ & $9,80 \mathrm{E}-04$ & $1,02 \mathrm{E}-03$ & $1,13 \mathrm{E}-03$ & $5,02 \mathrm{E}-04$ & $9,71 \mathrm{E}-04$ & $9,64 \mathrm{E}-04$ & $5,00 \mathrm{E}-04$ & $5,00 \mathrm{E}-04$ \\
Max & $9,86 \mathrm{E}-04$ & $9,80 \mathrm{E}-04$ & $1,02 \mathrm{E}-03$ & $1,13 \mathrm{E}-03$ & $5,02 \mathrm{E}-04$ & $9,71 \mathrm{E}-04$ & $9,64 \mathrm{E}-04$ & $5,00 \mathrm{E}-04$ & $5,00 \mathrm{E}-04$ \\
AVG & $9,86 \mathrm{E}-04$ & $9,80 \mathrm{E}-04$ & $1,02 \mathrm{E}-03$ & $1,13 \mathrm{E}-03$ & $5,02 \mathrm{E}-04$ & $9,71 \mathrm{E}-04$ & $9,64 \mathrm{E}-04$ & $5,00 \mathrm{E}-04$ & $5,00 \mathrm{E}-04$ \\
Std & $1,6 \mathrm{E}-02$ & $4,0 \mathrm{E}-03$ & $5,0 \mathrm{E}-02$ & $4,7 \mathrm{E}-02$ & $8,9 \mathrm{E}-04$ & $3,9 \mathrm{E}-02$ & $2,7 \mathrm{E}-02$ & $8,5 \mathrm{E}-04$ & $8,1 \mathrm{E}-04$ \\
Error & 8,45 & 8,32 & 13,19 & 11,56 & 5,45 & 9,36 & 8,10 & 5,40 & 5,25 \\
p-value & $8,60 \mathrm{E}-08$ & $6,09 \mathrm{E}-03$ & $6,80 \mathrm{E}-08$ & $1,06 \mathrm{E}-07$ & $5,31 \mathrm{E}-01$ & $7,41 \mathrm{E}-09$ & $4,60 \mathrm{E}-04$ & $6,61 \mathrm{E}-0$ & N/A \\
\hline
\end{tabular}

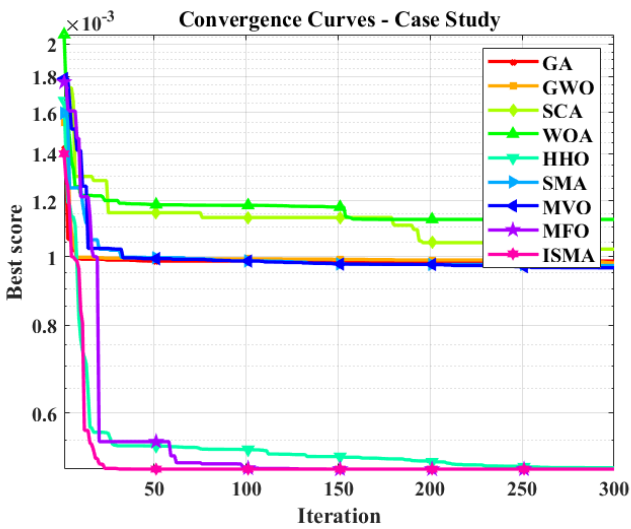

Figure 6. Convergence curves-case study 
Additionally, the proposed method has led to higher efficacy threshold. This result has been demonstrated through a benchmark with three selected standard classification data sets from the University of California at Irvine (UCI) machine learning repository [27]: sigmoid, cosine and sine. The dataset is shown in Table 3.

Table 3. Function approximation dataset

\begin{tabular}{ccc}
\hline Function approximation dataset & Training samples & Test samples \\
\hline Sigmoid: $y=1 /\left(1+e^{x}\right)$ & $61: x \in[3: 0.1: 3]$ & $121: x \in[3: 0.05: 3]$ \\
Cosine: $y=(\cos (x \pi / 2))^{7}$ & $31: x \in[1.25: 0.05: 2.75]$ & $38: x \in[1.25: 0.04: 2.75]$ \\
Sine: $y=\sin (2 x)$ & $126: x \in[-2 \pi: 0.1: 2 \pi]$ & $252: x \in[-2 \pi: 0.05: 2 \pi]$ \\
\hline
\end{tabular}

\subsection{Sigmoid function}

The sigmoid dataset belongs to the interval $[-3,3]$ with increases of 0.1 which sum up the number of training data to 61 . The number of test samples is 121 , lying in the same range. The test errors in Table 4, convergence curve in Figure 7 highlights that the ISMA algorithm admit the higher approximate exactitude in addition to the fastest convergence rate.

Table 4. Sigmoid dataset results

\begin{tabular}{cccccccccc}
\hline & GA & GWO & SCA & WOA & HHO & SMA & MVO & MFO & ISMA \\
\hline Min & $8,9 \mathrm{E}-09$ & $8,6 \mathrm{E}-09$ & $1,3 \mathrm{E}-02$ & $1,7 \mathrm{E}-03$ & $3,9 \mathrm{E}-04$ & $2,9 \mathrm{E}-04$ & $3,7 \mathrm{E}-04$ & $4,0 \mathrm{E}-04$ & $5,0 \mathrm{E}-09$ \\
Max & $5,8 \mathrm{E}-02$ & $4,5 \mathrm{E}-02$ & $1,4 \mathrm{E}-01$ & $1,5 \mathrm{E}-01$ & $7,8 \mathrm{E}-03$ & $6,4 \mathrm{E}-02$ & $6,5 \mathrm{E}-02$ & $5,6 \mathrm{E}-03$ & $2,9 \mathrm{E}-03$ \\
AVG & $1,6 \mathrm{E}-02$ & $3,6 \mathrm{E}-03$ & $6,3 \mathrm{E}-02$ & $4,6 \mathrm{E}-02$ & $5,1 \mathrm{E}-03$ & $1,2 \mathrm{E}-02$ & $1,0 \mathrm{E}-02$ & $2,5 \mathrm{E}-03$ & $8,0 \mathrm{E}-04$ \\
Std & $1,6 \mathrm{E}-02$ & $1,0 \mathrm{E}-02$ & $3,0 \mathrm{E}-02$ & $4,7 \mathrm{E}-02$ & $2,9 \mathrm{E}-03$ & $1,9 \mathrm{E}-02$ & $1,7 \mathrm{E}-02$ & $1,4 \mathrm{E}-03$ & $7,3 \mathrm{E}-04$ \\
Error & 0,78 & 0,20 & 9,63 & 3,50 & 1,41 & 1,56 & 1,77 & 1,79 & 0,14 \\
p-value & $8,60 \mathrm{E}-08$ & $5,61 \mathrm{E}-01$ & $6,80 \mathrm{E}-08$ & $1,06 \mathrm{E}-07$ & $2,60 \mathrm{E}-06$ & $7,41 \mathrm{E}-09$ & $4,60 \mathrm{E}-04$ & $9,28 \mathrm{E}-09$ & N/A \\
\hline
\end{tabular}

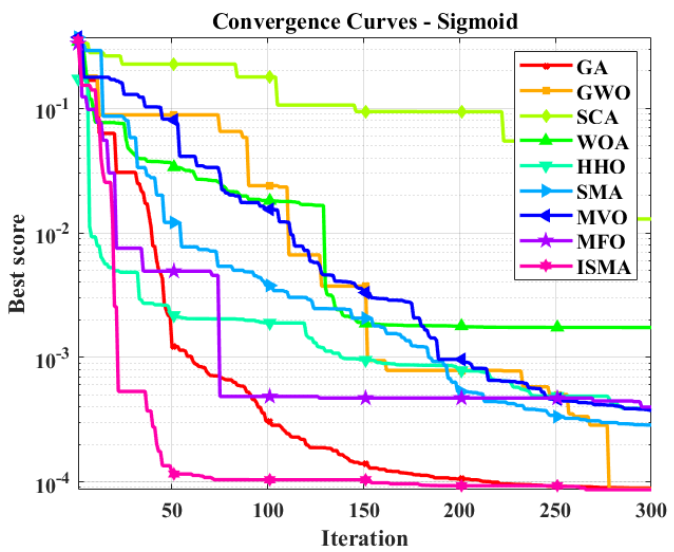

Figure 7. Convergence curves-sigmoid dataset

\subsection{Cosine function}

The cosine dataset belongs to the interval $[1.25,2.75]$ with increases of 0.05 , therefore the amount of training data is 31 . The number of test samples is 38 , lying in the same range. The test errors in Table 5, convergence and curve in Figure 8 highlights that the ISMA algorithm admit the higher approximate exactitude in addition to the fastest convergence rate.

Table 5. Cosine dataset results

\begin{tabular}{cccccccccc}
\hline & GA & GWO & SCA & WOA & HHO & SMA & MVO & MFO & ISMA \\
\hline Min & $3,8 \mathrm{E}-04$ & $2,1 \mathrm{E}-04$ & $2,9 \mathrm{E}-03$ & $3,1 \mathrm{E}-03$ & $2,1 \mathrm{E}-03$ & $1,7 \mathrm{E}-04$ & $6,7 \mathrm{E}-04$ & $1,3 \mathrm{E}-02$ & $1,2 \mathrm{E}-04$ \\
Max & $4,3 \mathrm{E}-03$ & $2,0 \mathrm{E}-03$ & $1,4 \mathrm{E}-02$ & $6,8 \mathrm{E}-02$ & $1,3 \mathrm{E}-01$ & $2,5 \mathrm{E}-03$ & $2,4 \mathrm{E}-03$ & $1,3 \mathrm{E}-01$ & $1,2 \mathrm{E}-03$ \\
AVG & $1,4 \mathrm{E}-03$ & $6,8 \mathrm{E}-04$ & $6,6 \mathrm{E}-03$ & $2,0 \mathrm{E}-02$ & $4,4 \mathrm{E}-02$ & $8,2 \mathrm{E}-04$ & $1,6 \mathrm{E}-03$ & $8,0 \mathrm{E}-02$ & $6,0 \mathrm{E}-04$ \\
Std & $8,8 \mathrm{E}-04$ & $4,6 \mathrm{E}-04$ & $3,1 \mathrm{E}-03$ & $2,0 \mathrm{E}-02$ & $4,9 \mathrm{E}-02$ & $6,7 \mathrm{E}-04$ & $4,9 \mathrm{E}-04$ & $3,9 \mathrm{E}-02$ & $3,3 \mathrm{E}-04$ \\
Error & 0,79 & 0,94 & 1,43 & 1,81 & 1,42 & 0,65 & 0,92 & 3,56 & 0,44 \\
p-value & $1,20 \mathrm{E}-01$ & $1,58 \mathrm{E}-08$ & $6,80 \mathrm{E}-06$ & $6,80 \mathrm{E}-06$ & $1,23 \mathrm{E}-07$ & $2,22 \mathrm{E}-04$ & $5,17 \mathrm{E}-08$ & $6,80 \mathrm{E}-06$ & $\mathrm{~N} / \mathrm{A}$ \\
\hline
\end{tabular}




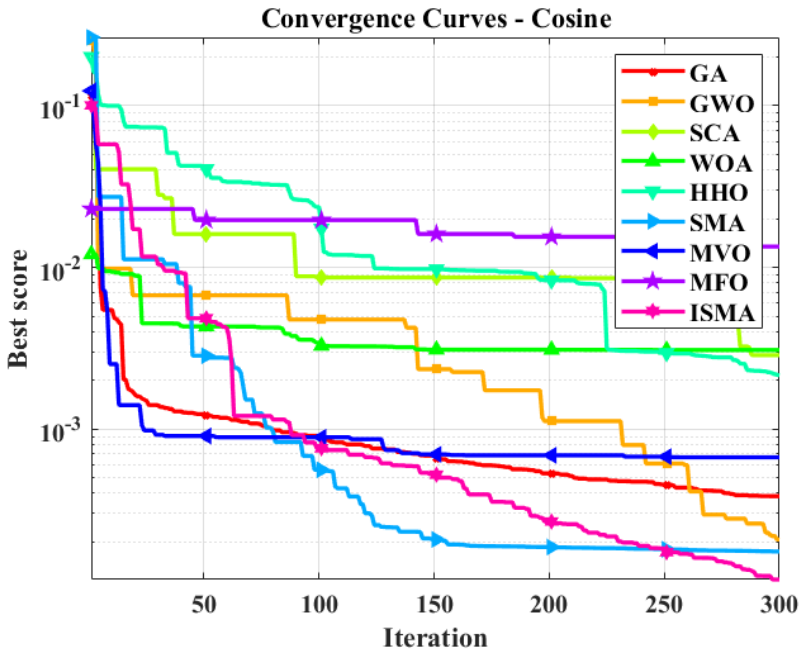

Figure 8. Convergence curves-cosine dataset

\subsection{Sine function}

The sine dataset belongs to the interval $[-2 \pi, 2 \pi]$ with increases of 0.1 , therefore the amount of training data is 126 . The number of test samples is 256 , lying in the same range. The test errors in Table 6 , convergence and curve in Figure 9 highlights that the ISMA algorithm admit the higher approximate exactitude in addition to the fastest convergence rate.

Table 6. Sine dataset results

\begin{tabular}{cccccccccc}
\hline & GA & GWO & SCA & WOA & HHO & SMA & MVO & MFO & ISMA \\
\hline Min & $8,0 \mathrm{E}-02$ & $6,1 \mathrm{E}-02$ & $1,4 \mathrm{E}-01$ & $1,1 \mathrm{E}-01$ & $1,1 \mathrm{E}-01$ & $4,9 \mathrm{E}-02$ & $1,6 \mathrm{E}-02$ & $1,1 \mathrm{E}-01$ & $4,4 \mathrm{E}-02$ \\
Max & $2,9 \mathrm{E}-01$ & $1,2 \mathrm{E}-01$ & $2,8 \mathrm{E}-01$ & $2,9 \mathrm{E}-01$ & $1,2 \mathrm{E}-01$ & $2,8 \mathrm{E}-01$ & $2,2 \mathrm{E}-01$ & $1,2 \mathrm{E}-01$ & $2,2 \mathrm{E}-01$ \\
AVG & $1,5 \mathrm{E}-01$ & $1,2 \mathrm{E}-01$ & $2,0 \mathrm{E}-01$ & $2,1 \mathrm{E}-01$ & $1,2 \mathrm{E}-01$ & $1,3 \mathrm{E}-01$ & $1,2 \mathrm{E}-01$ & $1,2 \mathrm{E}-01$ & $1,2 \mathrm{E}-01$ \\
Std & $5,9 \mathrm{E}-02$ & $5,0 \mathrm{E}-02$ & $3,6 \mathrm{E}-02$ & $5,4 \mathrm{E}-02$ & $2,3 \mathrm{E}-03$ & $5,5 \mathrm{E}-02$ & $6,3 \mathrm{E}-02$ & $2,5 \mathrm{E}-03$ & $2,4 \mathrm{E}-03$ \\
Error & 112,50 & 71,50 & 175,80 & 168,80 & 177,80 & 147,30 & 136,54 & 178,12 & 28,90 \\
p-value & $4,8 \mathrm{E}-02$ & $1,6 \mathrm{E}-02$ & $6,08 \mathrm{E}-06$ & $1,20 \mathrm{E}-08$ & $8,35 \mathrm{E}-03$ & $5,61 \mathrm{E}-02$ & $6,17 \mathrm{E}-03$ & $4,16 \mathrm{E}-04$ & N/A \\
\hline
\end{tabular}

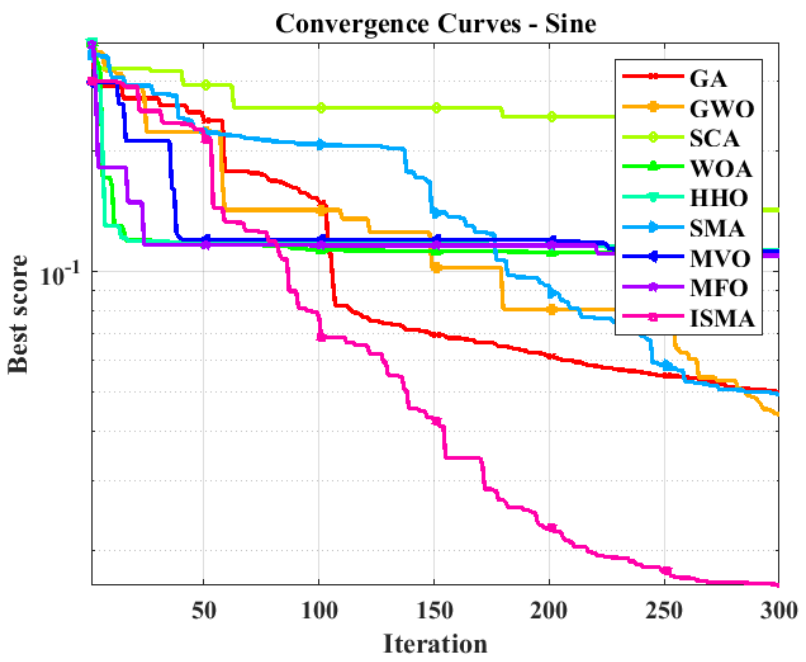

Figure 9. Convergence curves-sine dataset 


\subsection{Results discussion}

From statistical standpoint, the proposed ISMA shows high exploratory behavior to reach a superior local optima avoidance during FNNs training process within all datasets. The improved MSE algorithm is reflected by the important avoidance level of local optima. The mathematical formulation of the ISMA algorithm reports that, at each iteration, a fraction of the worst solutions is replaced by others while using the so-called Levy flight that forces the slim mould's to make random steps far from/towards the best slim mould. During FNNs training process, the search space's exploration supports to find out various FNN's structures. Besides, the position update of the population portion's rest is ensured by using a new mechanism based on APSO. Based on this analysis, the stagnation issue within local optima can be resolved through this approach in spite of being on exploitation process according to the ISMA algorithm. Nevertheless, during FNNs training and for every dataset, the search space is updated. In this regard, the outcomes of this study highlight that during the optimization process, in spite of the high exploration attitude of the evolutionary algorithms, the local optima avoidance is highly required for FNN training. Hence, the obtained results demonstrate the ISMA's effectiveness in this field. In other words, this study suggests the ISMA algorithm implementation in hybrid intelligent optimization endeavors, for example, FNNs training. Firstly, due to its high exploratory behavior to reach a superior local optima avoidance during FNNs training process. Secondly, one further important cause raised by the greatest exploitative behavior to converge quickly and for multiple datasets towards the global optimum.

\section{CONCLUSION}

This article contributes with a study to assess the operations' performance in the physical internet supply chain network. By contrast to other techniques, the approach of the artificial neural network has a major role to enhance the performance of the weaker performers to reach a multiple forthcoming efficiency ratings. The presented framework is established according to two steps basis. In the first step, the indicators influencing our system in addition to the performance measures are defined. Then, the efficiency scores are determined with the new approach involving the SMA in order to train the FNN. Subsequently, a performance evaluation has been conducted considering 100 Moroccan companies to highlight the efficacy of the established framework. Besides, the proposed ISMA-based trainer was applied to three standard approximation function datasets (sigmoid, cosine, and sine). For verification endeavors, night recent metaheuristic optimization trainers have been used to compare against the ISMA-FNN algorithm results. The results sustain that the proposed approach has confirmed its effectiveness to train FNNs. First of all, for FNNs, the ISMA-FNN algorithm has a major capacity to avoid the local optima. Furtermore, the accuracy for weights and biases optimal values is very prominent. In this paper, a global performane analysis has been realiased to highlight the related performance prons and cons of the other algorithms. In fact, for exploration process, the swarm-based algorithms' weakness has been recognized, as against the ISMA. For the upcoming studies, two valuable areas can be explored. According to FNNs, it is worthy to define the optimal number for hidden nodes, layers in addition to other structural parameter. Besides, Fine-tuning of this algorithm is a pledge of success also.

\section{REFERENCES}

[1] R. Bhagwat and M. K. Sharma, "Performance measurement of supply chain management using the analytical hierarchy process," Production Planning \& Control, vol. 18, no. 8, pp. 666-680, 2007, doi: 10.1080/09537280701614407.

[2] P. Taticchi, K. Balachandran, and F. Tonelli, "Performance measurement and management systems: state of the art, guidelines for design and challenges," Measuring Business Excellence, vol. 16, no. 2, pp. 41-54, 2012, doi: $10.1108 / 13683041211230311$.

[3] W. D. Cook and L. M. Seiford, "Data envelopment analysis (dea)-thirty years on," European journal of operational research, vol. 192, no. 1, pp. 1-17, 2009, doi: 10.1016/j.ejor.2008.01.032.

[4] P. W. Bauer, "Recent developments in the econometric estimation of frontiers," Journal of econometrics, vol. 46, no. 1-2, pp. 39-56, 1990, doi: 10.1016/0304-4076(90)90046-V.

[5] M. H. Hassoun, Fundamentals of artificial neural networks, Cambridge, MA, USA: MIT press, 1995.

[6] G. Dorffner, "Neural networks for time series processing," in Neural network world, 1996, doi: 10.1.1.45.5697.

[7] S. Ghosh-Dastidar and H. Adeli, "Spiking neural networks," International journal of neural systems, vol. 19, no. 4, pp. 295-308, 2009, doi: 10.1142/S0129065709002002.

[8] G. Bebis and M. Georgiopoulos, "Feed-forward neural networks," IEEE Potentials, vol. 13, no. 4, pp. 27-31, 1994, doi: $10.1109 / 45.329294$. 
[9] D. E. Rumelhart, G. E. Hinton, and R. J. Williams, "Learning representations by back-propagating errors," nature, vol. 323 , no. 6088 , pp. 533-536, 1986, doi: 10.1038/323533a0.

[10] P. A. Kowalski and S. Łukasik, "Training neural networks with krill herd algorithm," Neural Processing Letters, vol. 44, no. 1, pp. 5-17, 2016, doi: 10.1007/s11063-015-9463-0.

[11] H. Faris, I. Aljarah, and S. Mirjalili, "Training feedforward neural networks using multi-verse optimizer for binary classification problems," Applied Intelligence, vol. 45, no. 2, pp. 322-332, 2016, doi: 10.1007/s10489-016-0767-1.

[12] D. H. Wolpert and W. G. Macready, "No free lunch theorems for optimization," IEEE transactions on evolutionary computation, vol. 1, no. 1, pp. 67-82, 1997, doi: 10.1109/4235.585893.

[13] S. Li, H. Chen, M. Wang, A. A. Heidari, and S. Mirjalili, "Slime mould algorithm: A new method for stochastic optimization," Future Generation Computer Systems, vol. 111, pp. 300-323, 2020, doi: 10.1016/j.future.2020.03.055.

[14] J. Zhu, Quantitative models for performance evaluation and benchmarking: data envelopment analysis with spreadsheets, vol. 213, New York: Springer, 2014

[15] E. P. de Lima, S. E. G. da Costa, and A. R. de Faria, "Taking operations strategy into practice: developing a process for defining priorities and performance measures," International journal of production economics, vol. 122, no. 1, pp. 403-418, 2009, doi: 10.1016/j.ijpe.2009.06.022.

[16] X.-S. Yang, Nature-inspired metaheuristic algorithms, Luniver press, 2010.

[17] A. H. Gandomi, G. J. Yun, X.-S. Yang, and S. Talatahari, "Chaos-enhanced accelerated particle swarm optimization," Communications in Nonlinear Science and Numerical Simulation, vol. 18, no. 2, pp. 327-340, 2013, doi: 10.1016/j.cnsns.2012.07.017.

[18] R. Hecht-Nielsen, "Kolmogorov's mapping neural network existence theorem," in Proceedings of the international conference on Neural Networks, vol. 3, IEEE Press New York, 1987, pp. 11-14.

[19] J.-R. Zhang, J. Zhang, T.-M. Lok, and M. R. Lyu, "A hybrid particle swarm optimization-back-propagation algorithm for feedforward neural network training," Applied mathematics and computation, vol. 185, no. 2, pp. 1026-1037, 2007, doi: 10.1016/j.amc.2006.07.025.

[20] J. H. Holland, "Genetic algorithms," Scientific american, vol. 267, no. 1, pp. 66-73, 1992.

[21] S. Mirjalili, S. M. Mirjalili, and A. Lewis, "Grey wolf optimizer," Advances in engineering software, vol. 69, pp. 46-61, 2014, doi: 10.1016/j.advengsoft.2013.12.007.

[22] S. Mirjalili, "Sca: a sine cosine algorithm for solving optimization problems," Knowledge-based systems, vol. 96, pp. 120-133, 2016, doi: 10.1016/j.knosys.2015.12.022.

[23] S. Mirjalili and A. Lewis, "The whale optimization algorithm," Advances in engineering software, vol. 95, pp. 51-67, 2016, doi: 10.1016/j.advengsoft.2016.01.008.

[24] A. A. Heidari, S. Mirjalili, H. Faris, I. Aljarah, M. Mafarja, and H. Chen, "Harris hawks optimization: Algorithm and applications," Future generation computer systems, vol. 97, pp. 849-872, 2019, doi: 10.1016/j.future.2019.02.028.

[25] S. Mirjalili, S. M. Mirjalili, and A. Hatamlou, "Multi-verse optimizer: a nature-inspired algorithm for global optimization," Neural Computing and Applications, vol. 27, no. 2, pp. 495-513, 2016, doi: 10.1007/s00521-015-1870-7.

[26] S. Mirjalili, "Moth-flame optimization algorithm: A novel nature-inspired heuristic paradigm," Knowledge-based systems, vol. 89, pp. 228-249, 2015, doi: 10.1016/j.knosys.2015.07.006.

[27] A. Asuncion and D. Newman, "Uci machine learning repository," 2007. [Online]. Available: https://archive.ics.uci.edu/ml/index.php 